\title{
Affective auditory stimuli: Adaptation of the International Affective Digitized Sounds (IADS-2) for European Portuguese
}

\author{
Ana Paula Soares - Ana P. Pinheiro - Ana Costa • \\ Carla Sofia Frade • Montserrat Comesaña • Rita Pureza
}

Published online: 23 March 2013

(C) Psychonomic Society, Inc. 2013

\begin{abstract}
In this study, we present the normative values of the adaptation of the International Affective Digitized Sounds (IADS-2; Bradley \& Lang, 2007a) for European Portuguese (EP). The IADS-2 is a standardized database of 167 naturally occurring sounds that is widely used in the study of emotions. The sounds were rated by 300 college students who were native speakers of EP, in the three affective dimensions of valence, arousal, and dominance, by using the Self-Assessment Manikin (SAM). The aims of this adaptation were threefold: (1)to provide researchers with standardized and normatively rated affective sounds to be used with an EP population; (2)to investigate sex and cultural differences in the ratings of affective dimensions of auditory stimuli between EP and the American (Bradley \& Lang, 2007a) and Spanish (Fernández-Abascal et al., Psicothema 20:104-113 2008; Redondo, Fraga, Padrón, \& Piñeiro, Behavior Research Methods 40:784790 2008) standardizations; and (3)to promote research on auditory affective processing in Portugal. Our results indicated that the IADS-2 is a valid and useful database of digitized sounds for the study of emotions in a Portuguese context, allowing for comparisons of its results with those of other international studies that have used the same database for stimulus selection. The normative values of the EP
\end{abstract}

Electronic supplementary material The online version of this article (doi:10.3758/s13428-012-0310-1) contains supplementary material, which is available to authorized users.

A. P. Soares $(\bowtie) \cdot$ A. Costa $\cdot$ C. S. Frade $\cdot$ M. Comesaña R. Pureza

Human Cognition Lab, CIPsi, School of Psychology,

University of Minho, Campus de Gualtar,

4710-057 Braga, Portugal

e-mail: asoares@psi.uminho.pt

A. P. Pinheiro

Neuropsychophysiology Lab, CIPsi, School of Psychology,

University of Minho, Braga, Portugal adaptation of the IADS-2 database can be downloaded along with the online version of this article.

Keywords Affective auditory stimuli · IADS · Valence · Arousal $\cdot$ Dominance $\cdot$ European Portuguese

The detection of emotional salience in visual and auditory stimuli is a crucial aspect of human existence and social interactions (LeDoux, 1996; Phelps, 2006). However, research on affective processing has focused mainly on the processing of visual emotional stimuli such as emotional facial expressions, as well as emotional pictures and words, whereas less is known about affective processing in the auditory modality. In contrast with the static nature of pictures or words, sound changes dynamically throughout time, and thus the emotional meaning embedded in the acoustic stream - conveyed through the combination of acoustic cues such as fundamental frequency $\left(f_{0}\right)$, intensity, and duration (e.g., Banse \& Scherer, 1996; Juslin \& Laukka, 2003)needs to be continuously perceived, integrated, and interpreted (e.g., Shirmer \& Kotz, 2006). Differences between stimuli may lead to changes in the ways that people perceive, process, and experience affective information, which demands further investigation.

One of the major reasons why auditory stimuli are rarely used is possibly the lack of controlled and validated auditory stimuli that are adapted to the cultural context of the research participants, allowing for the comparison/replication of results from different research teams. This article addresses this issue, presenting the normative values of the European Portuguese (EP) adaptation of one of the most internationally used databases for the study of auditory affective processing: the International Affective Digitized Sounds (IADS; Bradley \& Lang, 1999b, 2007a). The EP adaptation of the IADS aims to provide researchers with a standardized research tool with the potential of promoting research on 
auditory affective processing in Portugal and that will allow for comparability of the results with other international studies.

The IADS is part of a system for emotional assessment developed by the Center for Emotion and Attention (University of Florida). This system also includes a database of words - the Affective Norms for English Words (ANEW: Bradley \& Lang, 1999a; already adapted for EP-see Soares, Comesaña, Pinheiro, Simões, \& Frade, 2012)and a database of pictures - the International Affective Picture System (IAPS; Lang, Bradley, \& Cuthbert, 1999; Lang, Bradley, \& Cuthbert, 2008; the adaptation for EP is in progress - see Soares, Comesaña, Pinheiro, Frade, Pureza, $\&$ Costa, 2011). The EP adaptation of these three emotional stimulus databases, which are based on the same emotional theoretical perspective and methodological procedure, represents a powerful tool for research on affective processing in any of these modalities (sounds, words, and pictures).

The original database of the IADS (Bradley \& Lang, 1999b), which was validated with an American English population, consisted of 111 digitally recorded sounds, each lasting for $6 \mathrm{~s}$ and characterized along the affective dimensions of valence, arousal, and dominance. The tridimensional assessment of the IADS stimuli follows a dimensional account of the emotions. On the basis of the semanticdifferential work developed by Osgood and associates (Osgood, Suci, \& Tannenbaum, 1957), Bradley and collaborators (Bradley, Codispoti, Cuthbert, \& Lang, 2001; Bradley \& Lang, 1994) proposed that emotional reactions and subjective experiences arise from the activation of two anatomically distinct motivational systems (defensive vs. appetitive) that have evolved to mediate the behaviors that sustain and protect life. The defensive system is primarily activated in contexts involving threat to the physical survival of the organism, with a basic behavioral repertoire that includes withdrawal or attack. Conversely, the appetitive system is activated in contexts that promote the organism's well-being and survival. These two systems account for two basic dimensions of emotion-valence and arousal-with valence indicating which system is active (defensive or appetitive), and emotional arousal reflecting the intensity of motivational activation. Hence, these constructs can be characterized by two bipolar dimensions representing the positivity-negativity (valence or pleasure) and the intensity (arousal or energy level) of a stimulus. Additionally, Bradley and Lang (1994) proposed a third dimension, dominance, which represents the level of control that a stimulus evokes. To measure these affective dimensions, the authors developed a nonverbal pictographic self-report measure: the Self-Assessment Manikin (SAM). IADS sounds were originally normalized using the SAM (see Fig. 1).

The second version of the IADS (IADS-2; Bradley \& Lang, 2007a), whose adaptation for EP is presented in this article, differs from the original version in the number of sounds included. The IADS-2 provides researchers with a standardized and reliable database of 167 naturally occurring sounds, which are associated with a wide number of contexts that can elicit a broad range of emotional reactions. These sounds can be related to humans (e.g., baby, erotic couple, boy laugh, giggling, female cough, man wheeze, or child abuse), animals (e.g., cat, puppy, growl, dog, seagull, or robin), objects (e.g., music box, typewriter, Polaroid camera, or doorbell), musical instruments (e.g., bugle, harp, guitar, or bagpipes), means of transport (e.g., jet, helicopter, train, plane crash, or bike wreck), or scenarios (e.g., tropical rainforest, country night, restaurant, or brook). Of note, the IADS database has already been adapted to Spanish populations (Fernández-Abascal et al., 2008; Redondo, Fraga, Padrón, \& Piñeiro, 2008), and beyond this threedimensional assessment, a categorical assessment of the IADS sounds within five discrete categories (happiness, sadness, fear, disgust, and anger) is also available with a sample of American English participants (see Stevenson \& James, 2008).

The relevance of this type of database is highlighted by evidence suggesting that nonverbal emotional sounds present several advantages over the speech stimuli commonly used in research, such as affective prosody in sentences or words (e.g., Brosch, Grandjean, Sander, \& Scherer, 2008; Liu, Pinheiro, Deng, Nestor, McCarley, \& Niznikiewicz, 2012; Pinheiro et al., 2013). Nonverbal emotional sounds seem to be the auditory analogue of emotional face expressions, representing "pure" vocal expressions of emotion (Sauter \& Eimer, 2010). As was pointed out by Scherer, Ladd, and Silverman (1984), the results of studies on affective prosody processing may be biased by the interaction between lexical and suprasegmental features of speech signals, including the affective meaning carried by prosody and the affective meaning carried by the semantic content of speech. Therefore, the use of naturalistic auditory stimuli, such as the sounds from the IADS database, may represent a suitable option for the study of affective processing, with minimal linguistic confounds.

Studies that have used IADS stimuli have revealed that auditory emotional stimuli activate the appetitive and defensive motivational systems similarly to the way that pictures do. In terms of autonomic responses, Bradley and Lang (2000), provided evidence that listening to affective (pleasant and unpleasant) sounds elicits physiological responsessuch as the startle reflex, facial electromyographic activity (EMG), or heart rate - that are similar to those elicited by affective pictures. Partala and Surakka (2003) observed similar effects in terms of pupillary responses for both emotionally negative and positive auditory stimuli in comparison with neutral stimuli, a finding that was recently replicated by Tressoldi, Martinelli, Semenzato, and 
Fig. 1 Self-Assessment Manikins (SAMs) for valence, arousal, and dominance. The five graphical figures and the spaces between them define the 9-point scales used
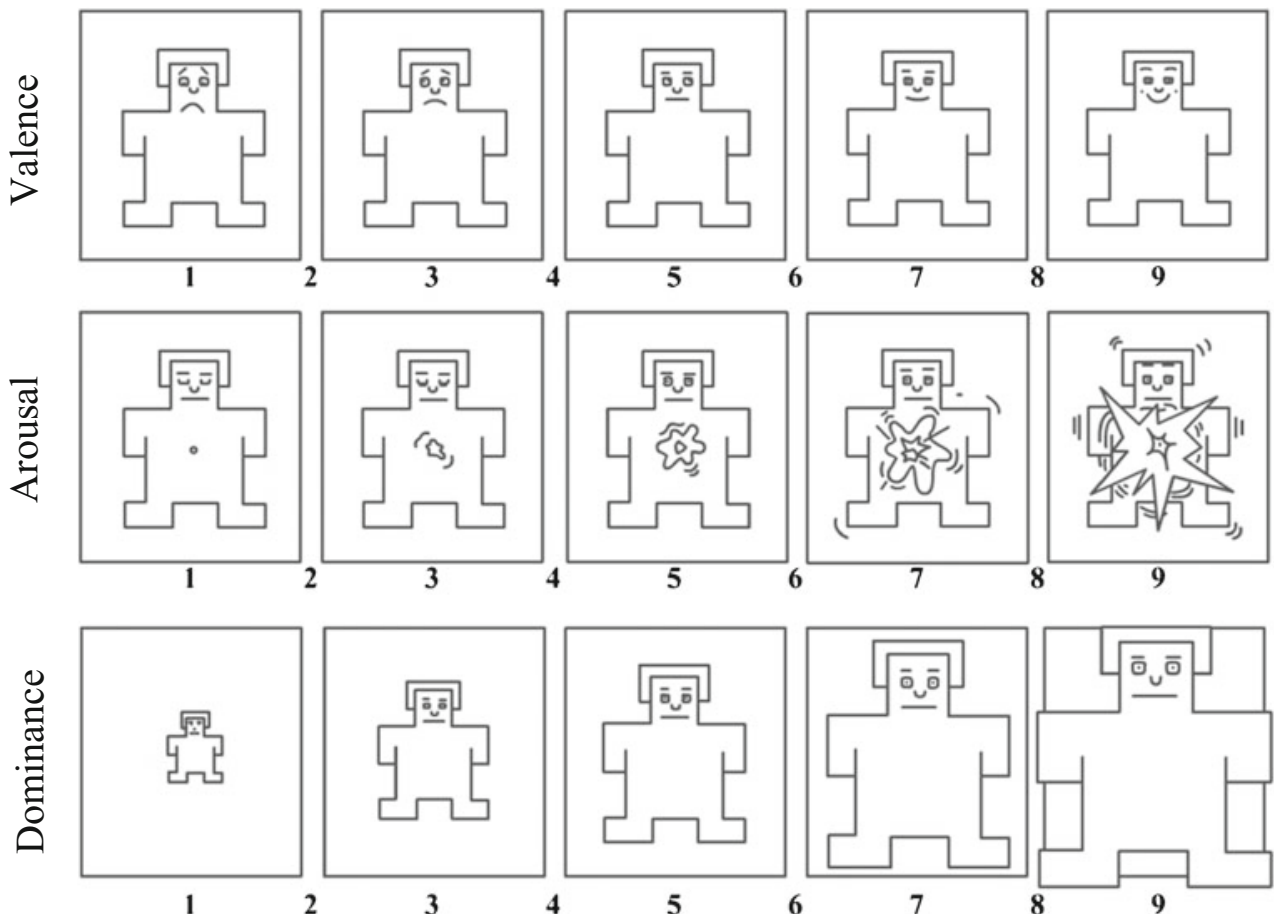

Cappato (2011) for alerting versus neutral sounds. The similar patterns of emotional reactivity to both affective sounds and pictures have supported the idea that responses to affective stimuli represent the activation of a common emotional system, rather than a modality-specific system (see Scharpf, Wendt, Lotze, \& Hamm, 2010).

Moreover, neuropsychological research has revealed differential processing of auditory stimuli with positive and negative valence, including stronger activation of the auditory cortex by positive (laughing) relative to negative (crying) sounds (e.g., Sander \& Scheich, 2001). In addition, greater left-hemisphere frontotemporal activation was reported in response to positively valenced auditory stimuli, while bilateral frontotemporal activation was observed in response to negatively valenced auditory stimuli (e.g., Alltenmüller, Schurmann, Lim, \& Parlitz, 2002). The latter evidence supports a valence asymmetry hypothesis, suggesting that the left hemisphere is specialized for the processing of positive emotions, while the right hemisphere is specialized for the processing of negative emotions (e.g., Heilman, 1997). This asymmetry hypothesis, though, has not received unequivocal support (see, e.g., Royet et al., 2000).

A negativity bias in attention allocation to auditory stimuli has also been demonstrated. For example, Smith, Cacioppo, Larsen, and Chartrand (2003) showed that negative information captures attention more automatically than do positive stimuli, and also that positive and negative stimuli are differentiated in early stages of information processing (around $100 \mathrm{~ms}$ after stimulus onset). In self-report measures of emotion, negative stimuli also tended to be assessed using more extreme scores for arousal than did positive stimuli (e.g.,
Bradley et al., 2001; Cacioppo, Gardner, \& Berntson, 1997). Erotic voice stimuli seem to represent the only exception, rated as being as arousing as angry voice stimuli, especially if spoken by actors of the opposite rather than the same sex as the listener (e.g., Ethofer et al., 2007), and as being more arousing than other pleasant stimuli (e.g., Bradley, Codispoti, Cuthbert, \& Lang, 2001; Lang, Bradley, Fitzsimmons, Cuthbert, Scott, Moulder, \& Nangia, 1998; Lykins, Meana, \& Kambe, 2006).

Studies on auditory affective processing have also demonstrated sex differences in response to sounds (e.g., Gohier et al., in press; Lewis, Wightman, Brefczynski, Phinney, Binder, \& DeYoe, 2004; Schirmer, Striano, \& Friederici, 2005), which had previously been observed for pictures (e.g., Bradley, et al., 2001; Bradley \& Lang, 2007b; Lithari et al., 2010), showing a female advantage in decoding of the affective proprieties of stimuli. Some studies have pointed to the greater sensitivity of women to distinguish stimuli with negative valence, such as negative nonverbal human vocalizations (e.g., a scream; e.g., Gohier et al., in press). Moreover, studies on emotional prosody have shown that women use emotional prosody earlier than men do (e.g., Schirmer, Kotz, \& Friederici, 2002), integrating emotional prosody into word processing even when the emotional prosody is task-irrelevant (e.g., Schirmer \& Kotz, 2003).

In the present study, we aimed to collect normative ratings of valence, arousal, and dominance for the IADS-2 stimuli in an EP population. Given the abovementioned differences in the ways that males and females seem to perceive, process, and respond to emotional stimuli, we also investigated potential sex differences in ratings of the 
affective dimensions of auditory stimuli in the EP standardization. In line with the previous research that we described earlier, we expected to find increased reactivity to affective auditory stimuli in females relative to males, particularly for negatively valenced stimuli.

Since IADS data-set norms are already available for other languages, we additionally explored whether cross-cultural differences in sounds' affective ratings would be observed when comparing the EP adaptation presented in this article with the American (Bradley \& Lang, 2007a) and Spanish (Fernández-Abascal et al., 2008; Redondo et al., 2008) IADS standardizations, as was done for the recent adaptation of ANEW to EP (Soares et al., 2012). As Mesquita and Walker (2003) pointed out, emotions are biological as well as sociocultural phenomena. Therefore, cultural practices may foster culture-specific appraisal tendencies, giving rise to differences in the ways that individuals from different cultures respond to emotionally salient stimuli. Cultural differences in emotional processing involve beliefs and social practices that define and reinforce what is considered moral, imperative, and desirable within each culture, and this is expected to affect emotional outputs (see Mesquita \& Walker, 2003). For example, in the EP adaptation of ANEW (Soares et al., 2012), cross-cultural differences were observed in the ways that Portuguese, American, and Spanish individuals reacted to affective words, with a greater similarity in the ratings between the EP and Spanish standardizations than between the EP and American English standardizations. Greater geographic and cultural proximity was the reason evoked by the authors to explain these results. Thus, as in Soares et al. (2012), we expected that the geographic and cultural similarities between Portugal and Spain would lead to greater similarity in the ratings of affective sounds between the EP and Spanish standardizations than between the EP and American English standardizations.

\section{Method}

\section{Participants}

A total of 300 college students ( 254 women and 46 men) from the University of Minho, with ages from 17 to 45 years $(M=$ $21.27, S D=4.49$ ) participated in the study. Participants received course credit for their participation in the experiment. The total sample did not include participants whose responses demonstrated nondiscriminative ratings and suggested random ratings or inattention (e.g., choosing the same number for all sounds $-2 \%$ of participants) or whose nationality and native language were not EP (4.7\%). All participants had normal audition and had normal or corrected-to-normal visual acuity. The majority of the participants were right-handed (92\%).
Materials and procedure

The stimuli consisted of 167 sounds that are part of the IADS- 2 and that had been used by Bradley and Lang (2007a) in their normative study. The physical properties of these sounds were controlled to avoid clipping and were normalized in terms of intensity.

The ratings session was programmed with SuperLab Pro 4.5 (Cedrus Corp., San Pedro, CA). This allowed for the controlled presentation of both instructions and sounds. The 167 sounds were divided into three randomized blocks (Block 1 contained 56 sounds, Block 2 contained 56 sounds, and Block 3 included 55 sounds) in order to avoid fatigue. Thus, each participant rated only one of these three blocks, but we assured that, across blocks, each sound would be rated by at least 93 participants $\left(M_{\text {Block } 1}=99.1\right.$ participants, out of 86 females and 14 males; $M_{\text {Block2 }}=98.3$ participants, out of 82 females and 18 males; and $M_{\text {Block } 3}=98.8$ participants, out of 86 females and 14 males).

Data collection followed the procedure described by Bradley and Lang (1999b, 2007a). The experiment was run in groups in a laboratory setting (the groups did not exceed ten participants per experimental session) by using, as in the original American standardization, a paper-andpencil version of SAM. In the SAM scale, each affective dimension is represented by a series of graphical figures illustrating a range of values for each of the three affective dimensions measured. Figure 1 illustrates the SAM version used in the present study: It contains five graphical figures defining a 9-point scale for each dimension: valence (ranging from 1, unpleasant, to 9 , pleasant), arousal (ranging from 1, calm, to 9, aroused), and dominance (ranging from 1 , controlled, to 9, in control).

After providing informed consent, participants received a booklet that listed the numerical codes of the sounds presented in a given experimental block and were instructed in the use of the 9-point scale of the SAM. Three practice sounds that were not included in the database (baby laughter, water drop, and woman yelling) but that had the same characteristics as the experimental stimuli were used to illustrate the valence range of the IADS sounds and to allow practice making ratings using the SAM scales. After listening to each practice sound, participants were instructed to choose the scale number that better represented the way that they felt while they were listening to that sound, for each affective dimension (i.e., valence, arousal, and dominance). Any questions or doubts were answered. In the booklet, participants also provided sociodemographic (e.g., sex, age, nationality, lateralization, and auditory and visual acuity) and linguistic (e.g., native language and second language learned) information.

During the experimental session, participants were seated in front of a computer screen at a distance of approximately 
$60 \mathrm{~cm}$. Each participant was randomly assigned to one of the three experimental blocks. The presentation of sounds in each block was randomized per participant. The structure of a trial for any of the three blocks was the following: Before participants listened to each of the experimental sounds, the instruction Por favor avalie o próximo som na linha número_ ["Please rate the next sound in line number_"] appeared in the center of the screen (Arial font, 14point) for $5 \mathrm{~s}$. Following the instructions, participants searched that numerical code in their response sheet (which corresponded to the row in which that specific sound would be rated in the valence, arousal, and dominance affective dimensions). The sound was then presented for $6 \mathrm{~s}$ through headphones. Immediately after the sound presentation, participants saw in the center of the screen (Arial font, 14point) the instruction Por favor avalie o som nas três dimensões afectivas ["Please rate the sound in the three affective dimensions now"], signaling the beginning of the response time (15 s), after which the next stimulus would be presented. Each trial lasted $26 \mathrm{~s}$, and the entire procedure took approximately $30 \mathrm{~min}$. Figure 2 presents the sequence of events of the experimental procedure that was followed.

\section{Results and discussion}

The affective norms for valence, arousal, and dominance for the 167 auditory stimuli of the IADS-2 (Bradley \& Lang, 2007a) that represent the adaptation of the IADS for EP can be downloaded as supplemental materials with this article. The supplemental archive shows the mean values $(M)$ and standard deviations $(S D)$ for valence ( $V a l)$, arousal (Aro), and dominance (Dom) of each of the 167 sounds of the adaptation of the IADS-2 to EP, considering the total sample $(\mathrm{All})$ and the female $(\mathrm{Fem})$ and male $(\mathrm{Mal})$ subsamples separately. The sounds were organized according to their original number (Sound Number) in the IADS-2 database (Bradley \& Lang, 2007a). After the number of each sound, the original description is presented (Description), followed by its EP translation (EP Description).

In the following sections, we first present the distribution of the ratings in the bidimensional affective space of valence and arousal, based on the EP adaptation of the IADS database. We then explore sex differences in these ratings. Finally, we present data on cross-cultural differences between the EP adaptation of the IADS and the American (Bradley \& Lang, 2007a) and Spanish (Fernández-Abascal et al., 2008; Redondo et al., 2008) standardizations.

\section{EP ratings of IADS-2 stimuli}

Figure 3 illustrates the distribution of the 167 EP sound ratings (mean values) in the bidimensional affective space of valence and arousal.

The distribution presented in Fig. 3 fits the typical boomerang shape found by Bradley and Lang in both the first (1999b) and the second (2007a) editions of the IADS, as well as by Redondo et al. (2008) and Fernández-Abascal et al. (2008) in the Spanish standardizations of the first edition of the IADS database. As is demonstrated by inspection of Fig. 3, the mean scores for each of the 167 sounds of the IADS- 2 are distributed along two axes stretching from a

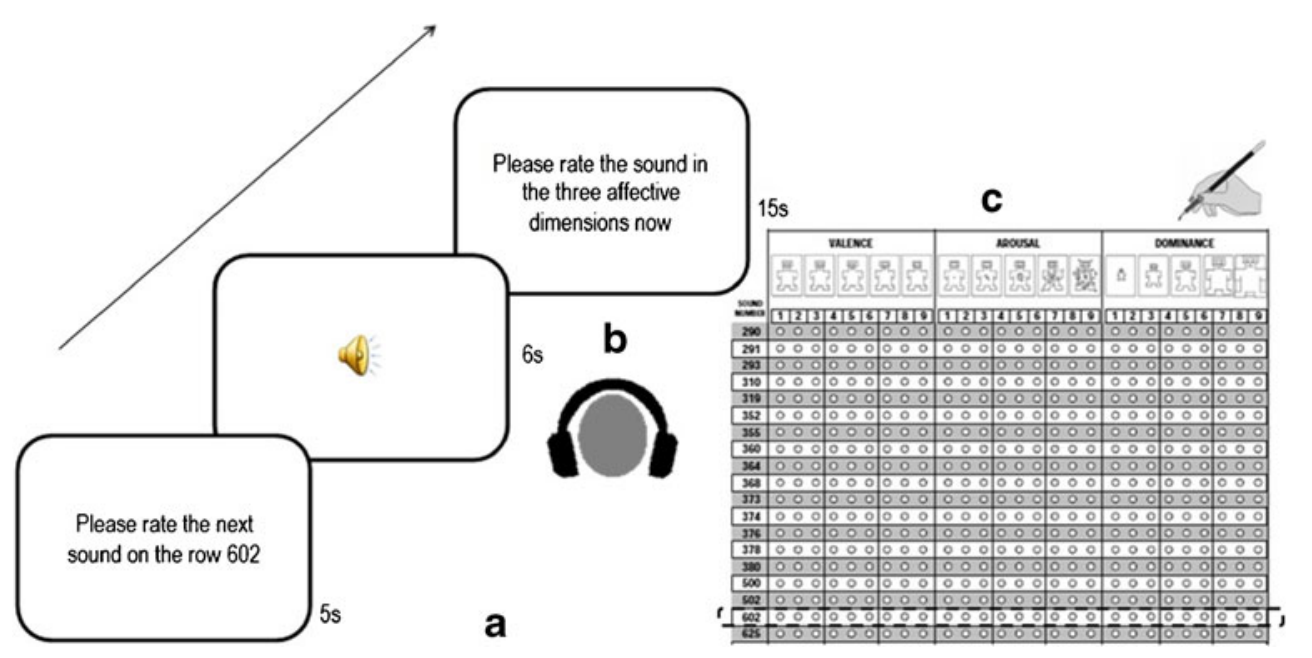

Fig. 2 Example of an experimental trial following the procedure adopted for ratings of the valence, arousal, and dominance of the IADS stimuli. (A)The participant would read the instruction Please rate the next sound in line number 602 and identify the row that corresponded to that number $(602)$ in the response sheet $(5 \mathrm{~s})$. (B)The participant would listen through headphones to the sound corresponding to the presented number $(6 \mathrm{~s})$. (C) The participant would read the instruction Please rate the sound in the three affective dimensions now, and rate the sound that he or she has just listened to along the three affective dimensions (valence, arousal, and dominance), using the SAM scales in the response sheet $(15 \mathrm{~s})$. This procedure was repeated for each of the sounds that was included in an experimental block 
Fig. 3 Distribution of overall mean values for the 167 sounds of the EP adaptation of the IADS-2 in the valence and arousal affective dimensions

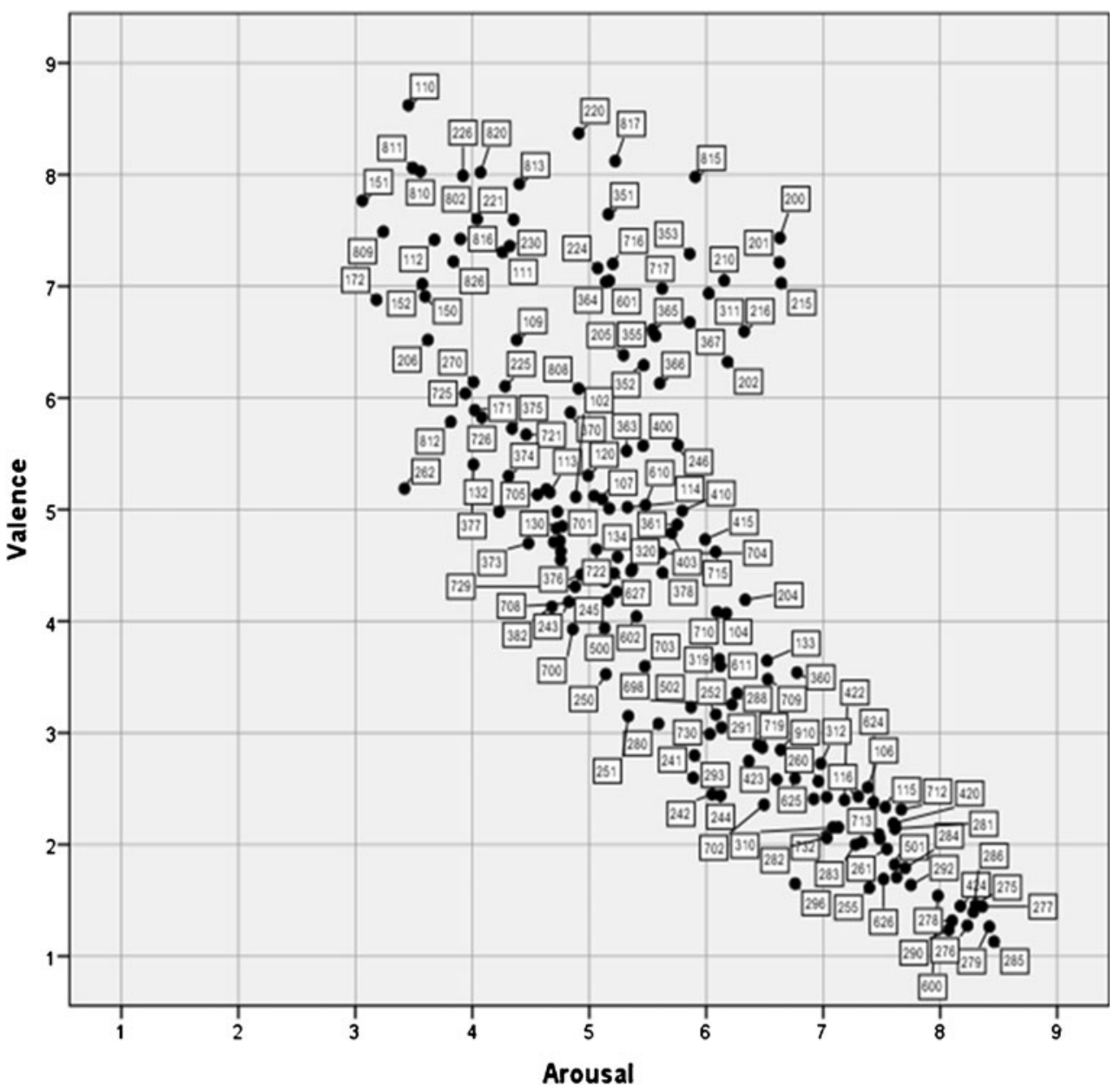

midpoint of neutrality in valence and arousal toward endpoints of either high-arousal positive or negative valence.

Thus, highly pleasant and highly unpleasant sounds were rated as being highly arousing, resulting in a quadratic correlation between valence and arousal that is more appropriate for the data than the linear correlation between the two dimensions. Even though the linear correlation between valence and arousal $(r=-.79, p<.001)$ in the EP standardization accounted for $62 \%$ of the variance, the quadratic correlation between the two dimensions $(r=.85, p<.001)$ accounted for $72 \%$ of the variance. This quadratic correlation was higher than the ones previously found in the American $(r=.74, p<.001$; see Bradley \& Lang, 2000) and Spanish $\left(r=.70, R^{2}=.472\right.$, $p<.001$; see Redondo et al., 2008) IADS standardizations.

Nevertheless, it is important to note that the association between valence and arousal for unpleasant sounds (i.e., sounds with valence ratings below 5 points, the midpoint of the 9-point scale used) is stronger $(r=-.91, p<.001)$ than the association between valence and arousal for pleasant sounds (i.e., sounds with valence ratings above 5), which did not reach statistical significance $(r=-.13, p=.30)$. Indeed, as can be seen in Fig. 3, most of the pleasant sounds (which are located in the upper half of the chart) were distributed along the arousal dimension $(M=4.75, S D=$ 0.92 , range $=3.06-6.64)$. This seems to indicate that valence is independent of arousal for positive sounds. However, for unpleasant sounds (which are located in the lower half of the chart), the ratings were more concentrated in the lower right quadrant of the chart $(M=6.39, S D=$ 1.13 , range $=4.23-8.46$ ), suggesting a strong negative relationship between valence and arousal for this type of sound. For example, Sounds 285 (Attack2), 290 (Fight1), and 279 (Attack1) were assessed as being the least positive sounds $\left(M_{\mathrm{val}}=1.13,1.24\right.$, and 1.26 , respectively) and as being the most arousing sounds $\left(M_{\text {aro }}=8.46,8.07\right.$, and 8.42, respectively) in this database. However, the same was not observed for pleasant sounds. For example, Sounds 110 (Baby), 220 (Boy laugh), and 817 (Bongos) were assessed as having the highest valence scores $\left(M_{\mathrm{val}}=8.62,8.37\right.$, and 8.12 , respectively), but not as having equivalent arousal scores $\left(M_{\text {aro }}=3.45,4.91\right.$, and 5.22, respectively). This seems to be the case only for erotic stimuli, such as Sounds 215 (EroticCouple), 200 (Erotic Couple), and 201 (EroticFemale), which simultaneously present high valence scores $\left(M_{\mathrm{val}}=7.03,7.43\right.$, and 7.21, respectively) and high arousal scores $\left(M_{\text {aro }}=6.64,6.63\right.$, and 6.63 , respectively). 
The finding that erotic sounds were assessed as having both higher valence and higher arousal scores confirms the "special" status of erotic sounds within the category of positively valenced stimuli and supports the notion that they are processed differently from other positive stimuli. Even though negative stimuli tend to elicit higher arousal levels than do positive stimuli (e.g., Bradley, et al., 2001; Cacioppo et al., 1997), some evidence suggests that erotic stimuli are the only type of positive stimuli that elicit arousal levels similar to those of negative stimuli (e.g., Bradley, et al., 2001; Lang et al., 1998), and that cause interference similar to that caused by aversive distractors (see, e.g., Most, Smith, Cooter, Levy, \& Zald, 2007; Yu, Hung, Tseng, Tzeng, Muggleton, \& Juan, 2012). The results obtained in this study demonstrate that the potential of erotic stimuli to elicit high levels of valence and arousal is not restricted to the visual modality (e.g., pictures), but also generalizes to the auditory domain of information processing.

Nonetheless, it is worth noting that in the EP adaptation of the IADS-2 it is easier to find unpleasant than pleasant sounds with high arousal scores, $t(165)=9.88, p<.001$. If we assume, as for valence, a cutoff value of 5 between classifications of arousing (above 5) and not arousing (below 5) sounds, it is possible to observe not only that a higher number of arousing sounds are classified as unpleasant rather than pleasant ( 98 vs. 69 , respectively), but also, for the unpleasant ones, that a higher number of sounds are classified as being high- (84) rather than low- (14) arousing sounds $\left[\chi^{2}(1)=33.34, p<.001\right]$. For the pleasant sounds, we observed the opposite pattern; that is, more sounds were assessed as being low-arousing (39) rather than high-arousing (30).

The negativity bias observed for the unpleasant stimuli in this study - which had previously been reported for pictures (e.g., Bradley et al., 2001; Bradley \& Lang, 2007b), sounds (e.g., Bradley \& Lang, 2000; Fernández-Abascal et al., 2008), and affective words (e.g., Bradley \& Lang, 1999a; Soares et al., 2012) - may hinder research using pleasant or unpleasant sounds when the manipulation of arousal level is intended. Nevertheless, besides this asymmetry, the dispersion of the results observed for both the valence (range $=1.13-8.62$ ) and arousal (range $=3.06-8.46$ ) dimensions will allow EP researchers to control and/or manipulate the affective properties of sounds according to the particular research interest.

\section{Sex differences in EP ratings of IADS-2 stimuli}

Table 1 presents means, standard deviations, and range values for each of the affective dimensions of valence, arousal, and dominance, for the subsamples of females and males separately.

In order to explore sex differences in the IADS-2 EP ratings, a multivariate analysis of variance (MANOVA) was conducted with Sex (females vs. males) and Sound
Valence (unpleasant vs. pleasant, classified on the basis of the ratings of the global sample) as between-subjects factors and the ratings of valence, arousal, and dominance as dependent variables. The analyses were thus run on items and not on subjects. Nonetheless, given the discrepancy between the numbers of males and females in our sample, we decided first to run the analysis with a random subsample of 46 females from the total sample of females $(N=254)$ to ensure that the number of females was equated with the number of males. This random subsample was obtained by creating a numbered list of all 254 female students and then by selecting 46 out of the 254 cases. In a second analysis, we computed the same statistical analysis with the total sample of females, in order to assess the stability of the results obtained. The results of the first MANOVA with 46 males and 46 females showed a main effect of sounds' valence in the affective dimensions of valence, $F(1,330)=700.06, p<.001, \eta^{2}=.68$, arousal, $F(1$, $330)=127.44, p<.001, \eta^{2}=.28$, and dominance, $F(1,330)=$ $346.38, p<.001, \eta^{2}=.51$, as well as a significant main effect of sex in the affective dimension of dominance, $F(1,330)=$ $4.81, p<.05, \eta^{2}=.02$. A significant interaction between sex and sounds' valences in the arousal, $F(1,330)=3.7, p<.05$, $\eta^{2}=.02$, and valence, $F(1,330)=2.92, p=.09, \eta^{2}=.01$ dimensions was also observed, although in the latter case, only at a marginally significant level.

The results of the second MANOVA, conducted with the total sample, replicated the results of the first analysis. As in the first MANOVA, we observed a main effect of sounds' valence in the affective dimensions of valence, $F(1,330)=755.50$, $p<.001, \eta^{2}=.69$, arousal, $F(1,330)=134.49, p<.001, \eta^{2}=$ .29 , and dominance, $F(1,330)=432.25, p<.001, \eta^{2}=.57$, even though the main effect of sex in the dominance dimension observed in the first analysis did not reach statistical significance here, $F(1,330)=2.59, p=.11, \eta^{2}=.01$. The interaction between sex and sounds' valences in the arousal dimension was also significant, $F(1,330)=4.47, p<.05, \eta^{2}=.02$, as well as the marginally significant interaction observed in the valence dimension, $F(1,330)=2.95, p=.08, \eta^{2}=.01$. The results observed with the first and second MANOVAs were consistent. Therefore, in spite of the discrepancy in the numbers of males and females in the adaptation of the IADS- 2 to EP, the sex differences observed were reliable. The normative values obtained with the total sample $(N=300)$ will thus be considered in the subsequent analyses.

The post-hoc Scheffé contrasts for the main effect of sounds' valence showed, as expected, that pleasant sounds were assessed as being more positive than unpleasant sounds $(p<.001)$. Also in line with the abovementioned findings, unpleasant sounds were assessed as being significantly more arousing than pleasant sounds $(p<.001)$, and additionally as having significantly lower dominance ratings $(p<.001)$. These findings confirm the previously reported 
Table 1 Means $(M)$, standard deviations $(S D)$, and range values for the 167 sound ratings of the EP adaptation of the IADS-2 for females and males in the three affective dimensions

\begin{tabular}{|c|c|c|c|c|c|c|}
\hline \multirow[t]{2}{*}{ Affective Dimensions } & \multicolumn{3}{|c|}{ Females } & \multicolumn{3}{|c|}{ Males } \\
\hline & $M$ & $S D$ & Range values & $M$ & $S D$ & Range values \\
\hline Valence & 4.52 & 2.09 & 7.68 & 4.69 & 1.86 & 6.88 \\
\hline Arousal & 5.73 & 1.35 & 5.38 & 5.64 & 1.34 & 6.01 \\
\hline Dominance & 4.78 & 1.53 & 5.84 & 4.98 & 1.41 & 5.64 \\
\hline
\end{tabular}

asymmetry between valence and arousal for pleasant and unpleasant stimuli (e.g., Bradley, et al., 2001; Bradley \& Lang, 2000, 2007b; Soares et al., 2012), as well as the fact that unpleasant stimuli tend to elicit subjective feelings of lower control (see LeDoux, 1996).

Furthermore the interaction between sex and sounds' valence for the arousal affective dimension revealed, as is illustrated in Fig. 4, that unpleasant sounds (i.e., those that scored below 5 in valence) were assessed as being more arousing by females than by males $(p<.05)$. However, pleasant sounds (i.e., those that scored above 5 in valence), especially erotic sounds (e.g., 200, 201, 202, and 215), tended to be assessed as being more arousing by males than by females. These findings of a negativity bias in the way that EP females rated IADS-2 sounds and a tendency for a positivity bias in the way that EP males rated the same sounds confirmed for the IADS-2 Portuguese adaptation a pattern that has been found in previous studies (e.g., Bradley, et al., 2001; Gohier et al., in press; Lithari et al., 2010; Schirmer et al., 2002, 2005). For example, Bradley and Lang (2007b) found that, while 30\% of females assessed IAPS unpleasant pictures as being more arousing (and only 15\% assessed pleasant pictures as being more
Fig. 4 Distribution of mean values for the 167 sounds of the EP adaptation of the IADS-2 in the valence and arousal affective dimensions, for males and females separately

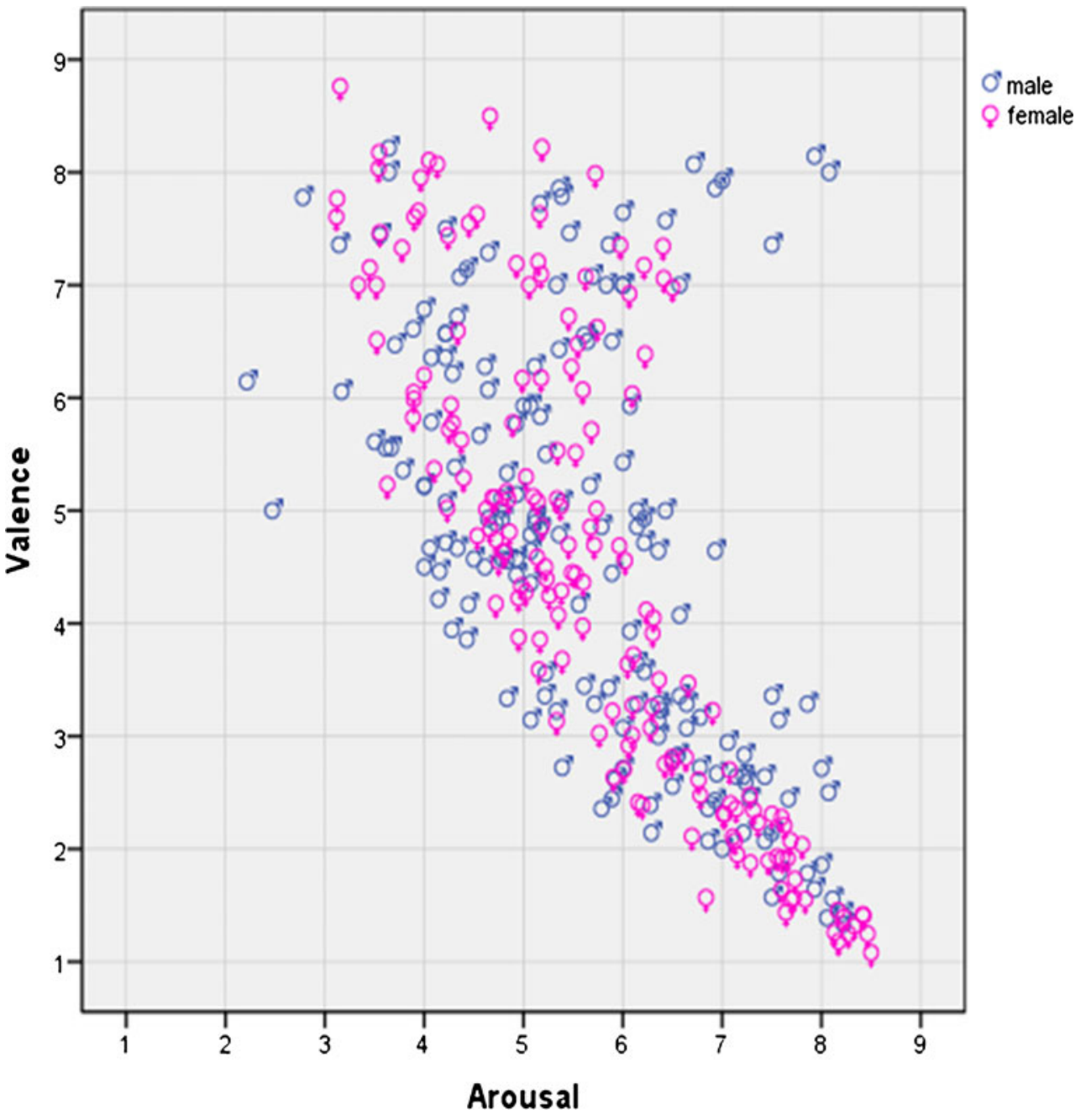


arousing), $40 \%$ of males assessed IAPS pleasant pictures as being more arousing (and only 15\% assessed unpleasant pictures as being more arousing).

It is also worth reemphasizing that within the category of pleasant stimuli, erotic sounds have a "special" status. Additionally, research has also demonstrated that the mechanisms associated with the processing of erotic stimuli seem to be distinct in males and females, with males showing higher peripheral physiological activation (e.g., Bradley, Codispoti, Sabatinelli, \& Lang, 2001) and also higher activation of limbic structures (e.g., Karama et al., 2002; Phelps, 2006; Royet et al., 2000) in response to erotic stimuli, relative to females. Males also show lower inhibitory control (e.g., Pessoa, 2009; Yu et al., 2012) in tasks involving the previous presentation of erotic stimuli. Our findings are consistent with this evidence.

Moreover, the marginally significant interaction between sex and sounds' valence for the valence affective dimension showed that females tend to produce more extreme scores when rating IADS stimuli; that is, they tend not only to assess unpleasant sounds as being more negative do males, but also to rate pleasant sounds as being more positive. These findings support our hypothesis and show that, as in previous studies (e.g., Bradley, et al., 2001; Lithari et al., 2010; Schirmer et al., 2002, 2005; Soares et al., 2012), Portuguese females tend to show higher emotional reactivity in response to affective sounds than do males.

However, the finding that the influence of sex on sound ratings was captured better when the two systems of appetitive versus defensive motivation were considered separately points to the need to attend to both systems of motivational mobilization when exploring sex differences. In line with existing studies (e.g., Bradley, et al., 2001; Lithari et al., 2010; Schirmer et al., 2002, 2005), sex differences were more pronounced for the defensive system. The fact that Portuguese females revealed more emotional reactivity toward negative sounds than did males may be explained by a complex interaction between the underlying biological processes and social and cultural responses. However, as was mentioned by Lithari et al., an evolutionary account may also explain this finding: The more rapid and stronger response of women to potentially dangerous stimuli (i.e., unpleasant and high-arousing stimuli) may have been useful for the effective nurturing of their offspring. The same kind of argument has also been proposed more recently to justify the differential processing associated with erotic stimuli within the category of positively valenced stimuli. Similarly to high-arousing negative stimuli, the reaction to erotic-type positive stimuli seems to reflect an important selection mechanism, given that these stimuli convey information that is of equal relevance for both survival and procreation for individuals (see Briggs \& Martin, 2009; Sakaki, Niki, \& Mather, 2012).

Therefore, the differences observed between males and females in the Portuguese adaptation of the IADS-2 suggest that these should be taken into account in the selection of sound stimuli when conducting research on affective processing with EP participants, especially if stimuli with negative valence are planned to be used.

\section{Cultural differences in EP ratings of the IADS-2 stimuli}

In order to explore cross-cultural differences in IADS ratings, we considered the four standardizations that have reported normative ratings for IADS stimuli: the American version of the IADS-2 (USA: Bradley \& Lang, 2007a), the two Spanish versions ( $\mathrm{SP}_{1}$ : Redondo et al., 2008; and $\mathrm{SP}_{2}$ : Fernández-Abascal et al., 2008), and the ratings obtained in the European Portuguese (EP) version. Given the existence of two Spanish adaptations published in the sameyear and the fact that they considered distinct numbers of sounds and distinct samples, we decided to integrate both standardizations in the analysis. The version of Redondo et al. considered all 111 sounds of the IADS original version (Bradley \& Lang, 1999b), which were rated by a sample of 159 college students, but the authors provided normative ratings only for the global sample, irrespective of sex. The second Spanish adaptation, published by Fernández-Abascal et al. (2008), included normative ratings of 110 sounds from the original IADS version (Bradley \& Lang, 1999b) rated by a wide sample of college students (1,136 females and 580 males), but contrary to Redondo et al., Fernández-Abascal et al. presented normative ratings not only for the global sample, but also for males and females separately.

Figure 5 illustrates the distribution of the mean values of IADS ratings in terms of the bidimensional affective space of valence and arousal for the American standardization (USA), the two Spanish adaptations ( $\mathrm{SP}_{1}$ and $\left.\mathrm{SP}_{2}\right)$, and the European Portuguese (EP) adaptation presented in this work.

The distribution of valence and arousal ratings presented in Fig. 5 indicates great overlap in the four standardizations, with all revealing the expected boomerang shape. In any of the presented standardizations, the quadratic relationship between valence and arousal was higher and captured more variance (for USA, $r=.64, R^{2}=.72, p<.001$; for $\mathrm{SP}_{1}$, $r=.69, R^{2}=.46, p<.001 ;$ for $\mathrm{SP}_{2}, r=.83, R^{2}=.70$, $p<.001$; for EP, $\left.r=.85, R^{2}=.72, p<.001\right)$ than did the corresponding pairwise linear correlations (for USA, $r=-.44, R^{2}=.19, p<.001$; for $\mathrm{SP}_{1}, r=-.46, R^{2}=$ $.21, p<.001$; for $\mathrm{SP}_{2}, r=-.75, R^{2}=.56, p<.001$; for $\left.\mathrm{EP}, r=-.79, R^{2}=.62, p<.001\right){ }^{1}$

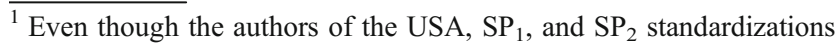
presented values of thelinear and quadratic correlations between valence and arousal (although in the $\mathrm{SP}_{2}$ standardization only linear correlation values were calculated), the values presented in this article were recalculated on the basis of the normative ratings included in their studies, since the comparison that we report in this work is with the second and not with the first edition of the IADS, as in those studies.
} 
Fig. 5 Distribution of mean values of IADS sounds ratings for the valence and arousal affective dimensions in the American (USA; Bradley \& Lang, 2007a), $\mathrm{SP}_{1}$ (Redondo et al., 2008), $\mathrm{SP}_{2}$ (FernándezAbascal et al., 2008), and European Portuguese (EP) versions

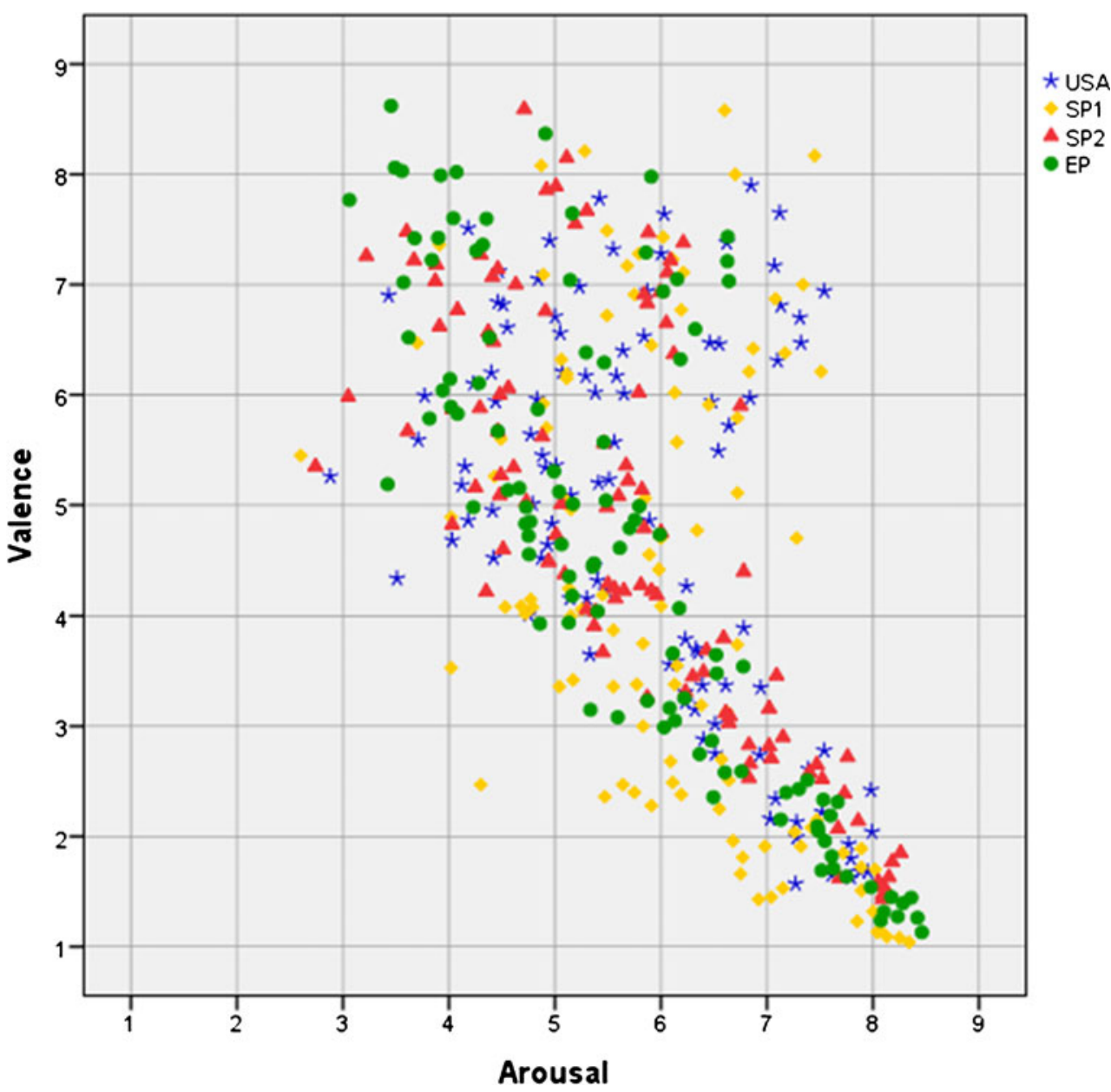

The similarities in the findings observed in the four standardizations may also be observed in Table 2, which presents the results of Pearson correlations between the ratings obtained in European Portuguese (EP), American English (USA), and Spanish $\left(\mathrm{SP}_{1}\right.$ and $\left.\mathrm{SP}_{2}\right)$ for each of the three affective dimensions. It is important to note that in the EPUSA correlational analysis, we considered the 167 sounds of IADS-2, while in the EP-SP ${ }_{1}$ correlational analysis, we considered only the 106 sounds that are common to both standardizations, and in the $\mathrm{EP}-\mathrm{SP}_{2}$ correlational analysis, we considered the 105 sounds that are common to both of those standardizations. In the specific case of $\mathrm{SP}_{1}$, we only considered correlations for the global sample, as mentioned above.

All correlations were positive and highly significant ( $p<$ .001 in all comparisons). ${ }^{2}$ It is worth noting that these

\footnotetext{
${ }^{2}$ Although the authors of the Spanish standardizations $\left(\mathrm{SP}_{1}\right.$ and $\left.\mathrm{SP}_{2}\right)$ presented Pearson correlations values between the affective ratings of their Spanish standardizations and USA, the values presented in this work were recalculated based on the basis of the normative ratings included in their studies, given that in the Spanish standardizations the authors considered the norms from the first and not the second edition of IADS as in this work.
}

correlations reveal stability not only for the global sample, but also for the subsamples of males and females separately. This suggests that IADS stimuli evoke similar emotional responses in individuals from different countries and cultures.

In order to explore with more detail the cross-cultural differences observed for each of the three affective dimensions of the IADS sound ratings across the four standardizations, we conducted a MANOVA with IADS Standardization (EP, USA, $\mathrm{SP}_{1}$, or $\mathrm{SP}_{2}$ ) and Sample Type (global sample, female subsample, or male subsample) as between-subjects factors and the affective dimensions of valence, arousal, and dominance as dependent variables. For this analysis, we only considered the global sample of the Spanish standardization published by Redondo et al. (2008). Table 3 presents means, standard deviations, and range sizes for each of the affective dimensions under analysis, for the global sample and for the subsamples of females and males separately.

The MANOVA showed a main effect of IADS standardization in the affective dimensions of valence, $F(3,1413)=$ $3.55, p<.05, \eta^{2}=.01$, arousal, $F(3,1413)=4.35, p<.01$, $\eta^{2}=.01$, and dominance, $F(3,1413)=6.53, p<.001, \eta^{2}=$ .02 , and a main effect of sample type in the dominance 
Table 2 Linear correlations between the European Portuguese (EP), American (USA, Bradley \& Lang, 2007a), and Spanish ( $\mathrm{SP}_{1}$, Redondo et al., 2008; $\mathrm{SP}_{2}$, Fernández-Abascal et al., 2008) IADS-2 sound ratings in the three affective dimensions for all participants, and for females and males separately

\begin{tabular}{|c|c|c|c|c|c|c|c|}
\hline \multirow{2}{*}{$\begin{array}{l}\text { Affective } \\
\text { Dimensions }\end{array}$} & \multicolumn{3}{|c|}{ All Participants } & \multicolumn{2}{|c|}{ Females } & \multicolumn{2}{|l|}{ Males } \\
\hline & USA & $\mathrm{SP}_{1}$ & $\mathrm{SP}_{2}$ & USA & $\mathrm{SP}_{2}$ & USA & $\mathrm{SP}_{2}$ \\
\hline Valence & $.93^{* *}$ & $.94^{* *}$ & $.95^{* *}$ & $.93^{* *}$ & $.94^{* *}$ & $.91^{* *}$ & $.95^{* *}$ \\
\hline Arousal & $.80^{* *}$ & $.78^{* *}$ & $.89^{* *}$ & $.79^{* *}$ & $.88^{* *}$ & $.82^{* *}$ & $.85^{* *}$ \\
\hline Dominance & $.92^{* *}$ & $.93^{* *}$ & $.93^{* *}$ & $.92^{* *}$ & $.92^{* *}$ & $.88^{* *}$ & $.88^{* *}$ \\
\hline
\end{tabular}

${ }^{* *} p<.001$

dimension, $F(2,1413)=3.29, p<.05, \eta^{2}=.01$. No interactions reached statistical significance $(p>.05)$.

The post-hoc Scheffé contrasts for the main effect of IADS standardization revealed that, even though the EP participants were not statistically different from American participants in the affective dimensions of valence, arousal, and dominance, they differed from the Spanish participants of the $\mathrm{SP}_{1}$ standardization, revealing lower scores in the affective dimension of arousal $(p<.001)$.

It is worth noting that Spanish participants of the $\mathrm{SP}_{1}$ standardization rated the IADS sounds with lower valence scores than did the participants of any of the other standardizations, although these differences only reached statistical significance when compared with participants of the $\mathrm{SP}_{2}(p<.05)$ and USA $(p<.05)$ standardizations. In the arousal dimension, Spanish participants of the $\mathrm{SP}_{1}$ standardization also differed from the participants of all other standardizations, rating IADS sounds with higher arousal scores. These differences were statistically significant not only for the comparison with EP, as already mentioned $(p<.001)$, but additionally with $\mathrm{SP}_{2}(p<$ $.001)$. Finally, in the dominance dimension, the Spanish participants of the $\mathrm{SP}_{1}$ standardization rated the IADS sounds with lower dominance scores than did those of any other standardization, although these differences only reached statistical significance when compared with the $\mathrm{SP}_{2}$ standardization $(p<$ .001). The MANOVA analysis also showed a main effect of sample type, revealing that in all IADS standardizations, males rated sound stimuli with higher dominance levels than were found among females $(p<.05)$, or even among the ratings of the global sample $(p<.05)$.

Together, these findings demonstrated similarities in the ratings obtained in the four standardizations of the IADS considered in this analysis. The only significant cross-cultural difference was with the Spanish standardization conducted by Redondo et al. (2008). The reduced sample size of the Redondo et al. standardization, and the associated limitations, may account for the differences observed.

Contrary to the hypothesis that geographic and cultural similarities between Portugal and Spain would lead to greater similarity in the IADS ratings between the EP-SP standardizations than between the EP-USA standardizations, as previously observed in the cross-cultural comparisons between the Spanish and the Portuguese ANEW ratings (see Soares et al., 2012). In fact, although research on cross-cultural differences in ratings of emotional sounds is scarce, a study of emotion recognition in vocal expressions conducted with participants from nine countries by Scherer, Banse, and Wallbott (2001) showed that, irrespective of cultural and linguistic origin,

Table 3 Mean, standard deviations (SD), and range values for the European Portuguese (EP), American (USA, Bradley \& Lang, Abascal et al., 2008) IADS sound ratings in the three affective dimensions for the global sample, and for females and males separately

2007a), and Spanish ( $\mathrm{SP}_{1}$, Redondo et al., 2008; $\mathrm{SP}_{2}$, Fernández-

\begin{tabular}{|c|c|c|c|c|c|c|c|c|c|c|}
\hline \multirow[t]{3}{*}{ Standardizations } & \multirow[b]{3}{*}{ Sample } & \multicolumn{9}{|c|}{ Affective Dimensions } \\
\hline & & \multicolumn{3}{|c|}{ Valence } & \multicolumn{3}{|c|}{ Arousal } & \multicolumn{3}{|c|}{ Dominance } \\
\hline & & $M$ & $S D$ & Range & $M$ & $S D$ & Range & $M$ & $S D$ & Range \\
\hline \multirow[t]{3}{*}{ EP } & All & 4.55 & 2.04 & 7.49 & 5.71 & 1.32 & 5.40 & 4.82 & 1.50 & 5.60 \\
\hline & Females & 4.52 & 2.09 & 7.68 & 5.73 & 1.35 & 5.38 & 4.78 & 1.53 & 5.84 \\
\hline & Males & 4.69 & 1.86 & 6.88 & 5.64 & 1.34 & 6.01 & 4.98 & 1.41 & 5.64 \\
\hline \multirow[t]{3}{*}{ USA } & All & 4.78 & 1.76 & 6.33 & 5.84 & 1.16 & 5.28 & 4.71 & 1.17 & 4.57 \\
\hline & Females & 4.72 & 1.92 & 6.77 & 5.89 & 1.20 & 5.70 & 4.59 & 1.28 & 5.26 \\
\hline & Males & 4.88 & 1.59 & 6.12 & 5.78 & 1.14 & 5.01 & 4.88 & 1.07 & 4.80 \\
\hline $\mathrm{SP}_{1}$ & All & 4.18 & 2.14 & 7.54 & 6.17 & 1.19 & 5.74 & 4.51 & 1.39 & 5.22 \\
\hline \multirow[t]{3}{*}{$\mathrm{SP}_{2}$} & All & 4.82 & 1.89 & 7.16 & 5.71 & 1.33 & 5.52 & 5.07 & 1.36 & 5.05 \\
\hline & Females & 4.80 & 1.90 & 7.22 & 5.76 & 1.34 & 5.56 & 5.01 & 1.38 & 5.14 \\
\hline & Males & 4.86 & 1.86 & 6.88 & 5.57 & 1.27 & 5.35 & 5.21 & 1.28 & 4.92 \\
\hline
\end{tabular}


participants were able to identify the vocally portrayed emotions with a high degree of accuracy. Since sounds carry emotional cues embedded in a continuously acoustic stream that are relatively independent of semantic content (based on the dynamic interplay of $f_{0}$, intensity, and duration; see Juslin \& Laukka, 2003; Schirmer \& Kotz, 2006), it is possible that their affective salience and meaning are accessed more automatically than is true for semantically mediated stimuli such as words (Scherer, Ladd, \& Silverman, 1984). Therefore, affective sounds may be more useful for the development of crosslinguistic studies and for the comparison of findings from studies on affective processing involving distinct research teams, countries, and cultures, avoiding the linguistic confounds that the use of words may imply (Sauter \& Eimer, 2010; Scherer et al., 1984).

\section{Conclusion}

In this study, we aimed to adapt the IADS-2 database (Bradley \& Lang, 2007a) to the EP context. The use of standardized stimuli is needed to effectively support current research on affective processing. Our results indicate that the IADS-2 is a valid and useful digitized sound database for the study of emotions in a European Portuguese context, allowing for comparisons of results with those from other international studies that have used the same database. As had previously been observed with both affective pictures (Lang et al., 1999, 2008) and words (Bradley \& Lang, 1999a; Soares et al., 2012), the results of the EP adaptation of IADS- 2 confirmed that the affective responses to sounds are organized between two motivational systems that regulate emotion expression (Bradley, et al., 2001; Bradley \& Lang, 2000).

Moreover, our findings revealed that males and females react differently to affective sounds, illustrating the importance of taking sex differences into account during the investigation of emotional processing. Therefore, it will be necessary to use norms derived from the adaptation of the IADS to EP for males and females separately in order to avoid confounding effects when doing research on auditory affective processing.

Finally, the cross-cultural analyses revealed strong stability in the results obtained for the three affective dimensions in the EP, American (Bradley \& Lang, 2007a), and Spanish (Fernández-Abascal et al., 2008; Redondo et al., 2008) standardizations, suggesting that sounds are less sensitive to cultural influences. Also, because of the underlying minimal influence of linguistic processing, IADS stimuli may represent an ideal option when conducting cross-cultural studies on affective processing.

However, even though no pronounced differences were found when comparing the four standardizations, it is important to note that research on auditory affective processing conducted in an EP context should use the normative values for the EP adaptation of the IADS-2. In spite of the similarities observed, the ratings for the EP, American, and Spanish standardizations are not exactly the same. Therefore, the norms reported in this study can better fit the sociocultural characteristics of the Portuguese population. Additionally, and contrary to what has been observed in other standardizations, the sex differences observed in the EP standardization of the IADS-2 strongly point to the need for researchers to use sex-adjusted norms in the development of studies within an EP context.

The use of the norms provided in this article can enable researchers to better control their experimental stimuli, compare results across studies, and combine stimuli with other normatively rated stimuli for the EP population, such as words (Soares et al., 2012). Therefore, the EP adaptation of the IADS-2 has the potential of becoming a useful tool for the development and internationalization of Portuguese research on affective auditory processing, both at unisensory and multisensory levels.

Author note This work is part of the research project "Procura Palavras (P-Pal): A Software Program for Deriving Objective and Subjective Psycholinguistic Indices for European Portuguese Words" (PTDC/PSI-PCO/104679/2008), funded by FCT (Fundação para a Ciência e Tecnologia), and FEDER (Fundo Europeu de Desenvolvimento Regional), through the European programs QREN (Quadro de Referência Estratégico Nacional), and COMPETE (Programa Operacional Factores de Competitividade).

\section{References}

Altenmüller, E., Schurmann, K., Lim, V. K., \& Parlitz, D. (2002). Hits to the left, flops to the right: Different emotions during listening to music are reflected in cortical lateralisation patterns. Neuropsychologia, 40, 2242-2256.

Banse, R., \& Scherer, K. R. (1996). Acoustic profiles in vocal emotion expression. Journal of Personality and Social Psychology, 70, 614-636.

Bradley, M. M., Codispoti, M., Cuthbert, B. N., \& Lang, P. J. (2001a). Emotion and motivation I: Defensive and appetitive reactions in picture processing. Emotion, 1, 276-298. doi:10.1037/15283542.1.3.276

Bradley, M. M., Codispoti, M., Sabatinelli, D., \& Lang, P. J. (2001b). Emotion and motivation II: Sex differences in picture processing. Emotion, 1, 300-319. doi:10.1037/1528-3542.1.3.300

Bradley, M. M., \& Lang, P. J. (1994). Measuring emotion: The Self-Assessment Manikin and the semantic differential. Journal of Behavioral Therapy and Experimental Psychiatry, 25, 4959.

Bradley, M. M., \& Lang, P. J. (1999a). Affective Norms for English Words (ANEW): Stimuli, instruction manual and affective ratings (Technical Report No. C-1). Gainesville, FL: University of Florida, Center for Research in Psychophysiology.

Bradley, M.M., \& Lang, P.J. (1999b). International Affective Digitized Sounds (IADS): Stimuli, instruction manual and affective ratings 
(Technical Report No. B-2). Gainesville, FL: University of Florida, Center for Research in Psychophysiology.

Bradley, M. M., \& Lang, P. J. (2000). Affective reactions to acoustic stimuli. Psychophysiology, 37, 204-215.

Bradley, M.M., \& Lang, P.J. (2007a). International Affective Digitized Sounds (2nd Edition; IADS-2): Affective ratings of sounds and instruction manual (Technical Report No. B-3). Gainesville, FL: University of Florida, NIMH Center for the Study of Emotion and Attention.

Bradley, M., \& Lang, P. J. (2007b). The International Affective Picture System (IAPS) in the study of emotion and attention. In J. A. Coan \& J. J. B. Allen (Eds.), Handbook of emotion elicitation and assessment (pp. 29-46). New York: Oxford University Press.

Briggs, K. E., \& Martin, F. H. (2009). Affective picture processing and motivational relevance: Arousal and valence effects on ERPs in an oddball task. International Journal of Psychophysiology, 72, 299-306.

Brosch, T., Grandjean, D., Sander, D., \& Scherer, K. R. (2008). Crossmodal emotional attention: Emotional voices modulate early stages of visual processing. Journal of Cognitive Neuroscience, $21,1670-1679$.

Cacioppo, J. T., Gardner, W. L., \& Berntson, G. G. (1997). Beyond bipolar conceptualizations and measures: The case of attitudes and evaluative space. Personality and Social Psychology Review, 1, 3-25.

Ethofer, T., Wiethoff, S., Anders, S., Kreifelts, B., Grodd, W., \& Wildgruber, D. (2007). The voices of seduction: Cross-gender effects in processing of erotic prosody. Social Cognitive and Affective Neuroscience, 2, 334-337.

Fernández-Abascal, E.G., Guerra, P., Martínez, F., Domínguez, F.J., Muñoz, M.A., Egea, D.A., ... Vila, J. (2008). El Sistema Internacional de Sonidos Afectivos (IADS): Adaptación española. Psicothema, 20, 104-113.

Gohier, B., Senior, C., Brittain, P.J., Lounes, N., El-Hage, W., Law, V., ... Surguladze, S.A. (in press). Gender differences in the sensitivity to negative stimuli: Cross-modal affective priming study. European Psychiatry. doi:10.1016/j.eurpsy.2011.06.007

Heilman, K. M. (1997). The neurobiology of emotional experience. Journal of Neuropsychiatry and Clinical Neuroscience, 9, 439-448.

Juslin, P. N., \& Laukka, P. (2003). Communication of emotions in vocal expression and music performance: Different channels, same code? Psychological Bulletin, 129, 770-814.

Karama, S., Lecours, A. R., Leroux, J. M., Bourgouin, P., Beaudoin, G., Joubert, S., et al. (2002). Areas of brain activation in males and females during viewing of erotic film excerpts. Human Brain Mapping, 16, 1-16.

Lang, P. J., Bradley, M. M., \& Cuthbert, B. N. (1999). International Affective Picture System (IAPS): Technical manual and affective ratings. Gainesville: University of Florida, Center for Research in Psychophysiology.

Lang, P.J., Bradley, M.M., \& Cuthbert, B.N. (2008). International Affective Picture System (IAPS): Affective ratings of pictures and instruction manual (Technical Report No. A-8). Gainesville, FL: University of Florida, NIMH Center for the Study of Emotion and Attention.

Lang, P. J., Bradley, M. M., Fitzsimmons, J. R., Cuthbert, B. N., Scott, J. D., Moulder, B., et al. (1998). Emotional arousal and activation of the visual cortex: An fMRI analysis. Psychophysiology, 35, 199-210.

Lewis, J. W., Wightman, F. L., Brefczynski, J. A., Phinney, R. E., Binder, J. R., \& DeYoe, E. A. (2004). Human brain regions involved in recognizing environmental sounds. Cerebral Cortex, 14, 1008-1021.

LeDoux, J. (1996). The emotional brain: The mysterious understanding of emotional life. New York: Simon \& Schuster.

Lithari, C., Frantzidis, C.A., Papadelis, C., Vivas, A.B., Klados, M.A., Kourtidou-Papadeli, C., ... Bamidis, P.D. (2010). Are females more responsive to emotional stimuli? A neurophysiological study across arousal and valence dimensions. Brain Topography, 23, 27-40.

Liu, T., Pinheiro, A. P., Deng, G., Nestor, P. G., McCarley, R. W., \& Niznikiewicz, M. A. (2012). Electrophysiological insights into processing nonverbal emotional vocalizations. NeuroReport, 23, 108

112

Lykins, A., Meana, M., \& Kambe, G. (2006). Detection of differential viewing patterns to erotic and non-erotic stimuli using eyetracking methodology. Archives of Sexual Behavior, 35, 569-575.

Mesquita, B., \& Walker, R. (2003). Cultural differences in emotions: A context for interpreting emotional experiences. Behaviour Research and Therapy, 41, 777-793.

Most, S. B., Smith, S. D., Cooter, A. B., Levy, B. N., \& Zald, D. H. (2007). The naked truth: Positive, arousing distractors impair rapid target detection. Cognition and Emotion, 21, 964-981.

Osgood, C. E., Suci, G., \& Tannenbaum, P. (1957). The measurement of meaning. Urbana: University of Illinois Press.

Partala, T., \& Surakka, V. (2003). Pupil size variation as an indication of affective processing. International Journal of Human Computer Studies, 59, 185-198.

Pessoa, L. (2009). How do emotion and motivation direct executive control? Trends in Cognitive Sciences, 13, 160-166. doi:10.1016/ j.tics.2009.01.006

Phelps, E. A. (2006). Emotion and cognition: Insights from studies of the human amygdala. Annual Review of Psychology, 57, 27-53.

Pinheiro, A.P., Del Re, E., Mezin, J., Nestor, P.G., Rauber, A., McCarley, R.W., et al. (2013). Sensory-based and higherorder operations contribute to abnormal emotional prosody processing in schizophrenia: An electrophysiological investigation. Psychological Medicine, 43(3), 603-618.

Redondo, J., Fraga, I., Padrón, I., \& Piñeiro, A. (2008). Affective ratings of sound stimuli. Behavior Research Methods, 40, 784790. doi:10.3758/BRM.40.3.784

Royet, J. P., Zald, D., Versace, R., Costes, N., Lavenne, F., Koenig, O., et al. (2000). Emotional responses to pleasant and unpleasant olfactory, visual, and auditory stimuli: A positron emission tomography study. Journal of Neuroscience, 20, 7752-7759.

Sakaki, M., Niki, K., \& Mather, M. (2012). Beyond arousal and valence: The importance of the biological versus social relevance of emotional stimuli. Cognitive, Affective, \& Behavioral Neuroscience, 12, 115-139.

Sander, K., \& Scheich, H. (2001). Auditory perception of laughing and crying activates human amygdala regardless of attentional state. Cognitive Brain Research, 12, 181-198.

Sauter, D. A., \& Eimer, M. (2010). Rapid detection of emotion from human vocalizations. Journal of Cognitive Neuroscience, 22, 474-481.

Scharpf, K. R., Wendt, J., Lotze, M., \& Hamm, A. O. (2010). The brain's relevance detection network operates independently of stimulus modality. Behavioral Brain Research, 210, 16-23.

Scherer, K. R., Banse, R., \& Wallbott, H. G. (2001). Emotion inferences from vocal expression correlate across languages and cultures. Journal of Cross-Cultural Psychology, 32, 76-92.

Scherer, K. R., Ladd, D. R., \& Silverman, K. (1984). Vocal cues to speaker affect: Testing two models. Journal of the Acoustical Society of America, 76, 1346-1356.

Schirmer, A., \& Kotz, S. A. (2003). ERP evidence for a gender specific Stroop effect in emotional speech. Journal of Cognitive Neuroscience, $15,1135-1148$

Schirmer, A., \& Kotz, S. A. (2006). Beyond the right hemisphere: Brain mechanisms mediating vocal emotional processing. Trends in Cognitive Sciences, 10, 24-30.

Schirmer, A., Kotz, S. A., \& Friederici, A. D. (2002). Sex differentiates the role of emotional prosody during word processing. Cognitive Brain Research, 14, 228-233. doi:10.1016/S0926-6410(02)00108-8

Schirmer, A., Striano, T., \& Friederici, A. D. (2005). Sex differences in the preattentive processing of vocal emotional expressions. NeuroReport, 16, 635-639.

Smith, N. K., Cacioppo, J. T., Larsen, J. T., \& Chartrand, T. L. (2003). May I have your attention, please: Electrocortical responses to positive and negative stimuli. Neuropsychologia, 41, 171-183. 
Soares, A. P., Comesaña, M., Pinheiro, A. P., Simões, A., \& Frade, C. S. (2012). The adaptation of the Affective Norms for English Words (ANEW) for European Portuguese. Behavior Research Methods, 44, 256-269. doi:10.3758/s13428-011-0131-7

Soares, A.P., Comesaña, M., Pinheiro, A.P., Frade, S., Pureza, R., \& Costa, A. (2011, March). Words, sounds and pictures: Affective norms of valence, arousal and dominance for European Portuguese [Palavras, sons e imagens: Normas afectivas de valência, activação e dominância para o português europeu]. Poster presented at the $6^{\circ}$ Encontro da Associação Portuguesa de Psicologia Experimental (APPE), University of Coimbra, Coimbra, Portugal.
Stevenson, R. A., \& James, T. W. (2008). Affective auditory stimuli: Characterization of the International Affective Digitized Sounds (IADS) by discrete emotional categories. Behavior Research Methods, 40, 315-321. doi:10.3758/BRM.40.1.315

Tressoldi, P.E., Martinelli, M., Semenzato, L., \& Cappato, S. (2011). Let your eyes predict: Prediction accuracy of pupillary responses to random alerting and neutral sounds. SAGE, 1-7.

Yu, J., Hung, D. L., Tseng, P., Tzeng, O. J. L., Muggleton, N. G., \& Juan, C. H. (2012). Sex differences in how erotic and painful stimuli impair inhibitory control. Cognition, 124, 251-255. 